\title{
Research on Wireless Communication Protocol of Internet of Things
}

\author{
Zixuan Zhao ${ }^{1, \text { a }}$
}

${ }^{1}$ Department of Internet of Things, Beijing University of Posts and Telecommunications, Beijing 100089, China

a email

Keywords: Wireless Communication Protocol, Internet of Things, Network Construction

\begin{abstract}
Internet of things is a new generation of information technology and an important part of the information industry. It is another leap-forward development. The most direct application of Internet of Things is wireless sensor network. The communication of the wireless sensor network needs the support of the basic communication protocol. This paper analyzes and summarizes the advantages and disadvantages of wireless transmission based on the analysis of current wireless transmission protocols.
\end{abstract}

\section{Introduction}

Internet of things is a new generation of information network technology and its rise will further promote the IT industry. However, due to the concept of things put forward a relatively short time, technology development has just started and in the international community has not a recognized architecture. As the research continues, a variety of Internet of Things architecture system is gradually being recognized. Technically, the Internet of Things includes four key areas: RFID (radio frequency identification), sensor network system, M2M (Machine to Machine) and "two networks integration." From the architecture point of view, the Internet of things can be divided into perception of the Internet layer, network transport layer and application layer.

Perceptual layer: The sensing layer is located at the bottom of the three-layer structure and the core layer of the wireless sensor network system. The basic unit of the sensing layer is the wireless sensor nodes, which are distributed in the network system. In the various data, it is to convert these data into electrical signals for the upper system control and analysis to provide raw data. It can and upper network communication, responsible for data transmission and command execution. It generally consists of a bar code reader, an RFID reader, a sensor, a video camera, and the like. The essence of the Internet of Things is to realize the "material connection" in the world. The sensing layer is the basic technology. It needs to capture and transform the physical quantity. In the network system, the wireless sensor node is mainly composed of processors, wireless transceiver modules and sensors, they can real-time on a variety of difficult environments and physical objects for detection and data acquisition, and this information through the wireless transceiver module to the upper, For the above transport layer and application layer to provide data can be processed, is the Internet of Things technology to achieve the foundation.

Network layer: the network layer is the middle layer of the entire Internet of Things architecture. It provides a gateway interface for the whole system. It is a data channel in the whole network system. It has a lot of data information in the Internet of Things system. It must rely on a powerful network for data fusion and transmission. The network layer in the network layer, the design techniques include: long-distance communications, wireless communications and related network security technologies. In the network layer, the design of long-distance communications, wireless communications and related network security technologies are important.

Application layer: The application layer is located at the top of the Internet of Things architecture. In the system, the sensing layer of the original data acquisition, and finally will focus on the application layer. It is mainly responsible for all the Internet of things in the original data analysis and processing, and data processing results through the man-machine interface to display in order to achieve better customer applications. Application layer can complete the main information processing, including information sharing, analysis and decision-making. It can be cross-regional, 
cross-level scheduling, in order to achieve maximum utilization of resources. In this layer will involve the database, cloud computing, user interface and other information processing technology.

Internet of Things is based on the evolution of the Internet technology, it and the Internet have some differences, the role of the Internet is the computer access to the same network system to achieve the sharing and integration of information resources, and Internet of things is the Internet Under the further development of the product is based on the Internet technology, combined with wireless communication technology for each object state of the amount of collection and transmission of the Internet, mobile communication network applications.

\section{Wireless Communication Node Design}

Wireless communication node is an important part of the Internet of Things system. It is applied in the Internet of Things architecture part of the perception layer, mainly distributed in a variety of application scenarios. In the node circuit design, the node must first consider the versatility and scalability. In the controller selection, it should be able to adapt to a variety of complex environments, the controller can not be affected by the environment caused by data collection and transmission performance degradation. In the Internet of Things system, the node covers a wide range, so the node in the cost, should be as low as possible.

Wireless communication nodes are mainly divided into two parts: advanced sensing nodes and basic sensing nodes. Advanced sensing node is composed of processor and RF module. The advanced sensing node has wide access capability, can realize the protocol conversion of heterogeneous network, and can configure and manage its own performance. A high-level aware node can perform self- In a number of devices to control the nodes and the upper sensing feedback, in the system in the bottom and upper coordinators. The main function of the device-aware node is to collect and transmit the physical quantities of the underlying devices, and communicate with the advanced sensing nodes. It mainly uses low-power single-chip microcomputer and short-distance communication module as the basis, and connects the sensors with different functions to realize Different information collection. A high-level aware node and a device-aware node form a one-to-many relationship.

\section{Wireless Transmission Protocol Classification}

WiFi is wireless Fidelity (wireless fidelity), also known as IEEE802.11b standard, the biggest advantage is the high transfer rate, up to 11mbps. WiFi wireless fidelity technology and Bluetooth technology, as belonging to the office and home use of short-range wireless technology, the use of the frequency band is near the $2.4 \mathrm{GHz}$ band, the band is currently useless license Of the wireless band, you can use the standard two 802.11ay and 802.11b, 802.11g is 802.11b inheritance.

General Packet Radio Service (GPRS), Packet Switched Mode with mobility management, and radio access technology. GPRS can be said to be a continuation of GSM. GPRS and the previous continuous channel transmission in different ways, is a packet (Packet) to transfer, so the user is borne by the cost of transmission data units, not the use of its entire channel, theoretically cheaper. GPRS transmission rate can be upgraded to 56 or even 114Kbps. GPRS technology is not suitable for smart furniture, mainly in the telecommunications network.

Infrared technology is also a wireless communication technology, can be wireless data transmission. Features: infrared transmission is point to point transmission, wireless, can not be too far away, to align the direction. It can not wear walls and obstacles, almost unable to control the progress of information transmission. 802.11 physical layer standard and is in addition to the use of $2.4 \mathrm{GHz}$ frequency of radio frequency. Also includes the relevant standards of infrared. IrDA (Infrared Data Association) Infrared Data Association, IrDA1.0 supports up to $115.2 \mathrm{kbps}$ communication rate. IrDA1.1 supports to 4Mbps. The technology is largely phased out, replaced by Bluetooth and newer technologies.

ZigBee is an emerging short-range, low-power, low-speed short-range wireless network technology. The basis for ZigBee is IEEE 802.15.4, a standard for the IEEE Wireless Personal Area 
Working Group. However, IEEE 802.15.4 only handles low-level MAC and physical layer protocols, so the ZigBee Alliance standardizes its network layer and API, while the Alliance is also responsible for its security protocols, application documentation and marketing. ZigBee Alliance was established in August 2001 by the British Invensys, Japan's Mitsubishi Electric, the United States Motorola, Philips Semiconductors and other companies in the Netherlands together. ZigBee and Bluetooth (Bluetooth), WiFi (Wireless LAN) belong to the $2.4 \mathrm{GHz}$ band IEEE standard network protocol, because of the different performance positioning their application is different. ZigBee's outstanding features are: ultra-low power, large network capacity, reliable data transmission, short delay, good security, low cost.

In the ZigBee technology, the security mechanism of the symmetric key is adopted. The key is generated, managed, stored, transmitted and updated by the network layer and the application layer according to the practical application requirements. Therefore, in the future of Internet of things, ZigBee technology is particularly important, and in the United States and other intelligent home networking has been widely used.

As an open global specification for wireless data and voice communications, Bluetooth is based on a low-cost, near-field wireless connection that establishes a special link between fixed and mobile device communication environments for short-range wireless transmission of data messages. The substance is to establish a common standard for the Radio Air Interface and its control software so that communications and computers can be further integrated so that portable devices produced by different manufacturers can, without wires or cables interconnected, Interoperability in the short-range range of interoperability, interoperability. Bluetooth is based on the IEEE802.11 standard for wireless LANs. The concept of "Plonkandplay" (somewhat similar to "plug and play") is applied, meaning that once a Bluetooth device has found another Bluetooth device, the connection can be established without any user setup, and thus can be interpreted as " Even with ". Bluetooth technology: low cost, low power consumption, small size, close communication, good security features. Bluetooth in the future development of things are to get a certain application, in office space, home intelligent home environment.

Radio Frequency Identification (RFID), also known as RFID (Radio Frequency Identification), is a communication technology that allows radio signals to identify specific targets and read and write related data without establishing a mechanism between the identification system and a specific target Or optical contact. The basic working principle of RFID technology: After the label enters the magnetic field, it receives the RF signal from the reader and sends out the product information stored in the chip (Passive Tag, Passive Tag or Passive Tag) with the energy obtained by the induced current, Send a signal frequency (Active Tag, active tags or active tags); reader reads the information and decoding, sent to the central information system for data processing. RFID is widely used in many fields at home and abroad, such as identification, monitoring and management, supermarkets, stores, shopping malls and other scenes to be a certain application.

\section{Conclusion}

Internet of Things is an important application of the new generation of information and communication technology, such as wireless sensor network. As the name implies, Internet of things is connected to the Internet. First, its core and reference is still the Internet, but on this basis and made a certain degree of expansion; Secondly, the client extends to all the objects in nature, so that everything in nature is Enabling information exchange. Internet of Things to achieve the use of a variety of existing technologies, such as short-distance communication, that is, ID, GPS, infrared detection, etc., using the Internet of things, can all things in this world and the Internet, so that the object Management and control more convenient.

With the continuous development of Internet of things technology and applications, wireless transmission protocol will usher in an unprecedented development in the future application of intelligent systems and will also show explosive growth. Understanding and master ZigBee, Bluetooth, Wi-Fi, RFID and other core technologies, the development of the corresponding interface and wireless communication products modular, wireless transmission protocol is a key 
technology of the development of things. It will also be the future development of the weight of things.

\section{References}

[1] Chui M, Löffler M, Roberts R. The internet of things [J]. McKinsey Quarterly, 2010, 2: 1-9.

[2] Mulligan G. The internet of things: Here now and coming soon [J]. IEEE internet computing, 2010, 14 (1) : 35-36.

[3] Akyildiz I.F. Wireless Sensor Networks: A Survey [J]. Computer Networks, 2002, 38: 393-422.

[4] Cullar D, Estrin D. Overview of Sensor Network [J]. Computer, 2004, 37 (8): 41-49.

[5] Demirkol I, Ersoy C, Alago F. MAC protocols for wireless sensor networks: a survey [J]. IEEE Communications Magazine 2006 44(4):115-121. 